\title{
Study on the Correlation between Nonalcoholic Fatty Liver and Metabolic Syndrome Based on Magnetic Resonance Diffusion-Weighted Imaging
}

\author{
Xiaoyu Liu ${ }^{(D)},{ }^{1}$ Zining Yao ${ }^{D},{ }^{2}$ Nan Li $\left(\mathbb{D},{ }^{3}\right.$ Kai Gao ${ }^{(D)},{ }^{1}$ and Sheng Zhu ${ }^{4}{ }^{4}$ \\ ${ }^{1}$ Department of Medical Imaging, The First District of Air Force Hangzhou Special Service Rehabilitation Center, \\ Hangzhou 310007, Zhejiang, China \\ ${ }^{2}$ Department of Laboratory Medicine, The Affiliated Hospital of Xiangnan University, Chenzhou 423000, Hunan, China \\ ${ }^{3}$ Department of Radiology, Binhai New Area Hospital of Traditional Chinese Medicine, Tianjin 300451, China \\ ${ }^{4}$ Department of Radiology, The Affiliated Hospital of Xiangnan University, Chenzhou, Hunan 423000, China
}

Correspondence should be addressed to Sheng Zhu; 2017800544@ctgu.edu.cn

Received 19 June 2021; Accepted 9 August 2021; Published 27 August 2021

Academic Editor: Gustavo Ramirez

Copyright (c) 2021 Xiaoyu Liu et al. This is an open access article distributed under the Creative Commons Attribution License, which permits unrestricted use, distribution, and reproduction in any medium, provided the original work is properly cited.

\begin{abstract}
This paper explores the relationship between magnetic resonance diffusion-weighted imaging of nonalcoholic fatty liver disease (NAFLD) and metabolic syndrome (MS), as well as the study of diffusion-weighted imaging, spectral imaging, and low density of nonalcoholic fatty liver correlation between serum concentration of lipoprotein cholesterol (LDL-C). This collection is from February 2018 to May 2018 in hospital as 39 cases of nonalcoholic fatty liver patients in the observation group and 39 patients in the control group with the same period of physical examination. All patients underwent magnetic resonance spectroscopy imaging, magnetic resonance diffusion-weighted imaging examination, and serum LDL-C test, compared the results of the two groups, and analysed the correlation between magnetic resonance spectroscopy imaging and magnetic resonance diffusionweighted imaging examination results and LDL-C. The results showed that the prevalence of NAFLD was $12.33 \%$, and the prevalence of NAFLD and MS was 3.47\%. Magnetic resonance spectroscopy observation parameter group and the control group had statistically significant difference when compared $(P<0.05)$. Difference was statistically significant $(P<0.01)$. In conclusion, nonalcoholic fatty liver DWI and MR spectroscopy is closely related to LDL-C targets, quantitatively and noninvasively reflects the body's fat metabolism, and can provide more extensive clinical diagnosis of nonalcoholic fatty liver. NAFLD is closely related to MS. The mutual aggravation and mutual promotion in the pathogenesis of the two should arouse enough attention and comprehensive prevention and treatment.
\end{abstract}

\section{Introduction}

Nonalcoholic fatty liver disease (NAFLD) refers to a group of liver diseases caused by the accumulation of fat, including liver steatosis, steatohepatitis, and combined inflammatory changes, fibrosis, and necrosis, with chronic inflammatory changes, necrosis, liver cirrhosis, and other diseases that progress to the end stage. NAFLD is a clinical disease with hepatocellular steatosis and fat accumulation as pathological features and no history of excessive drinking. NAFLD often coexists with metabolic syndrome (MS)-related diseases such as obesity, diabetes, and hyperlipidemia. At present, the correlation between NAFLD and MS has received widespread attention. The aim of this study was to understand the large number of healthy people in Chengdu and the prevalence of NAFLD and MS and analyse the relationship between NAFLD and MS. At present, magnetic resonance spectroscopy imaging technology is the only noninvasive imaging technique that can reflect the pathological and physiological changes of living tissues from the perspective of molecular metabolism. Currently, diffusion weighted imaging (DWI) is the only viable tissue that can detect the thermal motion of water molecules in non-invasive magnetic resonance imaging technology. In order to study the 
relationship between LDL-C in patients with nonalcoholic fatty liver MRS and DWI and low-density lipoprotein cholesterol, the relevant case data analysis is selected and researched. The report is as follows [1].

\section{Objects and Methods}

2.1. General Information. 39 patients with nonalcoholic fatty liver admitted to our hospital from February 2018 to May 2018 were used as the observation group, including 23 males and 16 females, aged 19-71 years, with an average age of $(58.67 \pm 11.26)$ years. After undergoing MRI and DWI examinations, the observation group patients were confirmed to be nonalcoholic fatty liver by multiple examinations, which is in accordance with the 2006 edition of "Diagnosis and treatment of nonalcoholic fatty liver disease" revised by the 2006 Chinese Medical Association Liver Society Fatty Liver and Alcoholic Hepatology. The relevant diagnostic criteria were based on the Chinese Medical Association Liver Society Fatty Liver and Alcoholic Hepatology, and 39 healthy people who underwent physical examination in our hospital during the same period were selected as the control group [2].

Diagnostic criteria are as follows: body mass index $(\mathrm{BMI})$ = body weight $(\mathrm{kg}) /$ height $(\mathrm{m})$. MS diagnostic criteria refers to the diagnostic criteria of the Chinese Diabetes Society (CDS) of MS and the recommended diagnostic criteria of MS. Those with 3 or all of the following 4 items are diagnosed with MS: (1) overweight and/ or obese: $\mathrm{BMI} \geq 25 \mathrm{~kg} / \mathrm{m}^{2}$; (2) hypertension: systolic/diastolic blood pressure $\geq 140 / 90 \mathrm{mmHg}$, including this blood pressure measurement is lower than this value but has a history of hypertension and currently taking antihypertensive drugs; (3) hyperglycemia: $F P G \geq 6.1 \mathrm{mmol} / \mathrm{L}$, including those whose blood glucose value is lower than this time but have a history of diabetes and are currently taking hypoglycaemic drugs; and (4) blood lipid disorder: fasting $\mathrm{TG} \geq 1.7 \mathrm{mmol} / \mathrm{L}$ and (or) HDL-C males $<0.9 \mathrm{mmol} / \mathrm{L}$; female $<1.0 \mathrm{mmol} / \mathrm{L}$.

\subsection{Method}

2.2.1. Instruments and Related Reagents. Magnetic resonance is produced by Siemens (Germany) model avanto1.5 T eight-channel phase-controlled surface coil, breathing gating, and MRS is processed with a randomly configured postprocessing software package (spectroscopy). The instrument used is Olympus automatic biochemical analyzer (OLYMPUS, Japan), model 2700. Low-density lipoprotein cholesterol measurement kit LDL-C (direct measurement method) was purchased from Beijing Leadman Biochemical Co., Ltd., with product standard number (YZB/Beijing 0538-2008).

2.2.2. Routine MRI. Each subject is trained in breathing before the examination to ensure that they breathe accurately and accurately, and the subject is told that the whole process takes about 30-40 minutes to obtain the best cooperation of the subject. All subjects arrange check in at 8-9 pm. Siemens avanto1.5 T superconducting magnetic resonance scanner with eight-channel phase-controlled surface coil is used. The subject is placed in a supine position, arms are raised, and the head is advanced. From the portal of the liver, a prestarvation zone is added above and below the scanning field to reduce the interference of the gas in the lungs, gastrointestinal tract, gastrointestinal motility, and cardiac pulsation. The fat suppression technology is used to eliminate the chemical shift forming artifacts. Breath-triggered technology is routinely used for scanning to minimize the effects of breathing movements [3]. The fast spin echo sequence of fat suppression horizontal axis and coronal is collected. T2WI parameter is as follows: TR/TE $=2000$ / $79 \mathrm{~ms}$. The T1WI parameter of the spin echo sequence of the horizontal axis position is as follows: TR/TE $=133$ / (2.4-6.15) ms. Fatty liver evaluation was performed using a double echo sequence with scrambled gradient echoes. The TE phase was $1.15 \mathrm{~ms}$, and the phase was the same; the $2.3 \mathrm{~ms}$ phase was the same, and the TR was $245 \mathrm{~ms}$.

2.2.3. $1 H-M R S$, DWI Scanning. Magnetic resonance $1 \mathrm{H}-$ MRS and DWI examinations use Siemens avanto1.5 T superconducting magnetic resonance instrument, eightchannel phase-controlled surface coil, and horizontal axis position and coronal position are used to locate $1 \mathrm{H}-\mathrm{MRS}$ and DWI regions of interest (ROI). In the right posterior lobe, try to avoid visible bile ducts, blood vessels, and calcified foci, necrotic tissue, and cystic areas (Figures 12).

1H-MRS inspection uses the monomer voxel positioning method-point resolution selection spectroscopy (PRESS) sequence which is TE50 ms, TR2000 ms, 8 times of excitation, voxels $20 \mathrm{~mm} \times 20 \mathrm{~mm} \times 20 \mathrm{~mm}$, adds saturation band around the ROI, so as to eliminate the surrounding tissue and respiratory movement the impact of the composition. The postprocessing of image data is completed in the postprocessing of the workstation. DWI check $b$ value is 0 , 50, 400, 800, and $1000 \mathrm{~s} / \mathrm{mm}^{2}$; scanning parameters are TR1600 $\mathrm{ms}$ and TE47 $\mathrm{ms}$, dispersion direction is ALL, layer spacing is $1 \mathrm{~mm}$, layer thickness is $5 \mathrm{~mm}$, matrix is $128 \times 128$, excitation is done twice, 13 layers are scanned, inhale deeply to exhale again to end-expiratory apnea to scan, the scan is completed within an average apnea, and the scanning time is $14 \mathrm{~s}$. Single voxel positioning method-point resolution is adopted to select the spectrum (PRESS). The sequence is TE50ms, TR2000ms, and 8 excitations, and the voxel is $20 \mathrm{~mm} \times 20 \mathrm{~mm} \times 20 \mathrm{~mm}$. The data obtained by the image is completed in the workstation. During DWI inspection, the $\mathrm{b}$ values are $0,50,400,800$, and $1000 \mathrm{~s} / \mathrm{mm} 2$; the scanning parameters are TE47ms and TR1600ms, the dispersion direction is ALL, the interlayer distance is $1 \mathrm{~mm}$, the layer thickness is $5 \mathrm{~mm}$, and the matrix is $128 \times 128$. Excites twice and scans 13 layers. The scan is completed within the average apnea, and the scan time is 14 seconds [4].

2.2.4. Image Postprocessing and Analysis. The data processing and display are processed using the postprocessing workstation software that comes with SINMENS at random. 


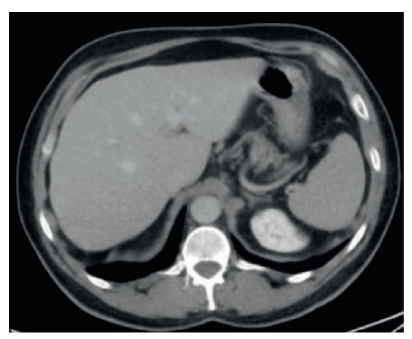

(a)

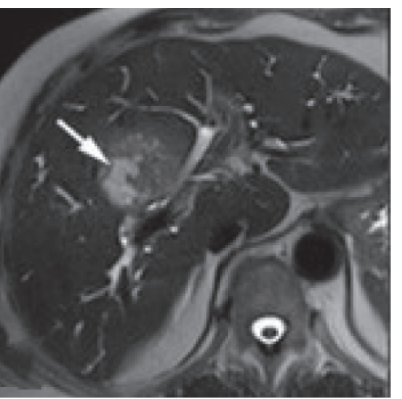

(a)

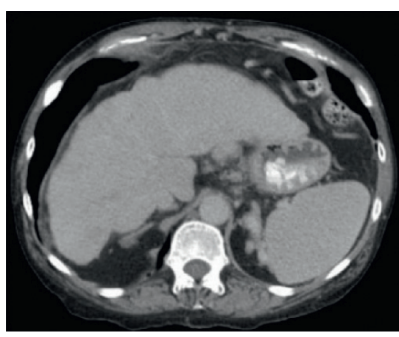

(b)

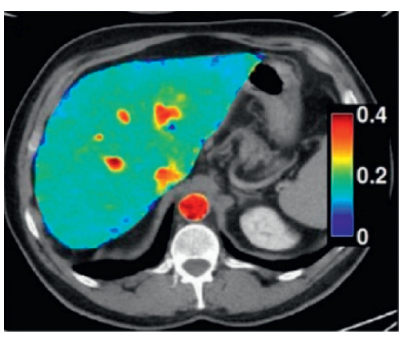

(c)

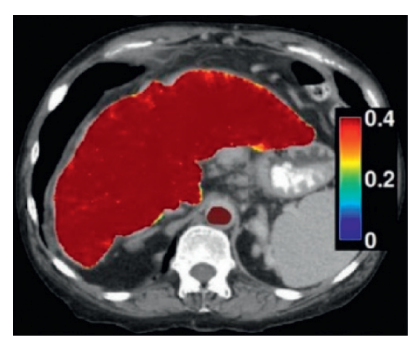

(d)

FIGURE 1: 1H-MRS imaging image of liver.

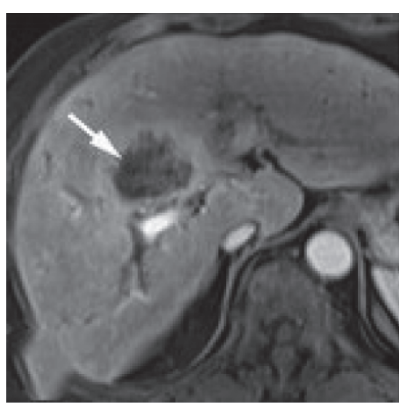

(b)

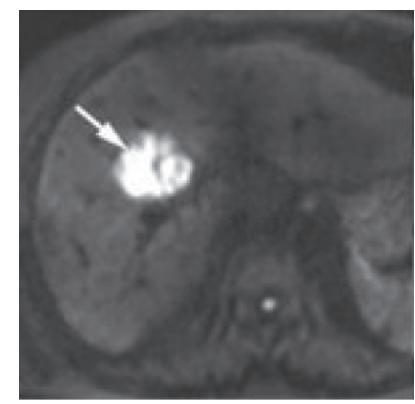

(c)

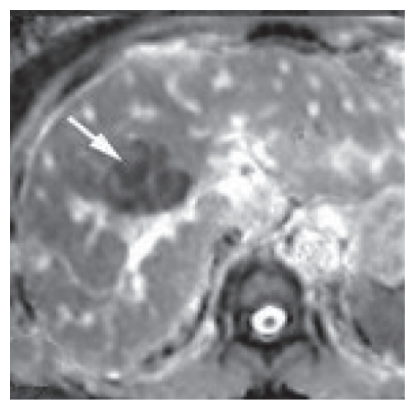

(d)

FIGURE 2: DWI imaging images of liver.

Spectral data use the Gaussian formula to perform Fourier transform in time and three-dimensional space and use the ratio of the area under the peak of the biochemical substance as its ratio. Data postprocessing steps are as follows. first, two deputy chief physicians with rich experience in abdominal imaging diagnosis performed phase correction on the confirmed nonalcoholic fatty liver patient spectrum line so that the baseline of metabolites was at the same level. The comparison was made between different metabolite contents more accurate; the fitted spectrum line and the machine automatically calculate the area under the peak of the metabolite, which is about $1.33 \mathrm{ppm}$ to get the lipid peak, and $4.9 \mathrm{ppm}$ to get the water peak. The relative ratio of the metabolite peak area is as follows: area under lipid peak/ (area under water peak + area under lipid peak) $\times 100 \%$, and the ratio is obtained as the ratio of fat to water [5]. In addition, the DWI and ADC maps were evaluated to determine the optimal $b$ value of the best image analysis for diffusion limitation. The data measurement was performed on the ADC image using Siemens' data measurement software package to directly measure the ADC value. During the measurement, three circular regions of the same size in the whole liver were taken to avoid large blood vessels, bile ducts, visible bile ducts, blood vessels and calcified lesions, necrotic tissue, and cystic changes. The area of interest is set by the investigator, and two deputy chief physicians evaluate whether it is reasonable and take the average value after the measurement.

Fourier transformation is an important milestone in the field of signal processing. It plays a very important role in the image processing art nursery rhyme and is widely used for image feature extraction, image enhancement and reduction, noise reduction, texture analysis, and so on. With wide range of applications in image processing, Fourier transform plays a very important role and is reflected in image analysis, image enhancement, image compression, and so on. Suppose $f(x, y)$ is the two-dimensional discrete space function, the two-dimensional Fourier transform of this function is defined as follows:

$$
F(u, v)=\sum_{x=0}^{M-1} \sum_{y=0}^{N-1} f(x, y) e^{-j 2 \pi((u x / M)+(v y / N))} .
$$

The definition of the inverse discrete Fourier transform is as follows:

$$
f(x, y)=\frac{1}{M N} \sum_{u=0}^{M-1} \sum_{v=0}^{N-1} F(u, v) e^{j 2 \pi((u x / M)+(v y / N))} .
$$

Assuming that $f(x, y)$ is a two-dimensional function in a discrete space, the two-dimensional discrete cosine transform of this function is defined as follows: 


$$
\begin{aligned}
& C(u, v)=a(u) a(v) \sum_{X=0}^{N-1} \sum_{y=0}^{N-1} f(x, y) \cos \left[\frac{(2 x+1) u \pi}{2 N}\right] \cos \left[\frac{(2 y+1) v \pi}{2 N}\right], \\
& f(x, y)=\sum_{u=0}^{N-1} \sum_{v=0}^{N-1} a(u) a(v) C(u, v) \cos \left[\frac{(2 x+1) u \pi}{2 N}\right] \cos \left[\frac{(2 y+1) v \pi}{2 N}\right] .
\end{aligned}
$$

After an image undergoes the Fourier transform, it changes from the spatial domain to the frequency domain, so we can use the signal processing method for frequency domain signals to process an image, for example, low-pass filtering the image. $F(u, v)$ is called the discrete Fourier transform coefficient of $f(x, y)$. This formula shows that the function $f(x, y)$ can be represented by the sum of countless complex exponential signals at different frequencies, while the amplitude and phase of the complex exponential signal at frequencies $\left(w_{1}, w_{2}\right)$ are $f\left(w_{1}, w_{2}\right)$.

Using the characteristics of Fourier transform to perform DTC transform, the image boundary after image conversion is folded to form an even function, and then the image is subjected to two-dimensional Fourier transform. When the cosine transform only contains terms, it is called the discrete cosine transform. Definition of two-dimensional discrete cosine transform DCT is assumed that the size of the matrix $A$ is $M \times N$.

$$
\begin{aligned}
& B_{p \cdot q}= \alpha_{p} \alpha_{q} \sum_{m-0}^{M-1} \sum_{n=0}^{N-1} A_{m n} \cos \frac{\pi(2 m+1) p}{2 M} \cos \frac{\pi(2 n+1) q}{2 N}, \\
& \alpha_{P}=\left\{\begin{array}{ll}
\frac{1}{\sqrt{M}}, & P=0, \\
\sqrt{\frac{2}{M}}, & 1 \leq P \leq M-1,
\end{array} \quad \alpha_{q}= \begin{cases}\frac{1}{\sqrt{N}}, & q=0, \\
\sqrt{\frac{2}{N}}, & 1 \leq q \leq N-1 .\end{cases} \right.
\end{aligned}
$$

Among them, $B_{p, q}$ is called the DCT coefficient of matrix $A$. In MATLAB, the subscript matrix form 1 rather than 0 , so the matrix elements of $A(1,1)$ and $B(1,1)$ MATLAB, respectively, correspond to the above definitions and values. DCT is a reversible transform, and inverse discrete cosine transform is defined as follows:

$$
\begin{aligned}
A_{m n} & =\sum_{p-0}^{M-1} \sum_{q-0}^{N-1} \alpha_{p} \alpha_{q} B_{p q} \cos \frac{\pi(2 m+1) p}{2 M} \cos \frac{\pi(2 n+1) q}{2 N}, \\
0 & \leq m \leq M-1,0 \leq n \leq N-1 .
\end{aligned}
$$

The meaning of the formula is that any $M \times N$ matrix $A$ can be expressed as a function of the sum of a series of the form as follows:

$$
\begin{aligned}
& \alpha_{p} \alpha_{q} B_{p q} \cos \frac{\pi(2 m+1) p}{2 M} \cos \frac{\pi(2 n+1) q}{2 N}, \\
& 0 \leq p \leq M-1,0 \leq q \leq N-1 .
\end{aligned}
$$

In this way, $B_{p, q}$ can be seen as a weight applied to each basis function. The discrete cosine transform can be calculated from the definition. However, such calculation is too large, which is very inconvenient in practical applications. So, we need to find a fast algorithm. Take the one-dimensional discrete cosine transform as a column to derive the fast algorithm.

$$
\begin{aligned}
F(u)= & \sqrt{\frac{2}{N}} \sum_{x=0}^{N-1} f(x) \cos \frac{(2 x+1) u \pi}{2 N} \\
& \sqrt{\frac{2}{N}} \operatorname{Re}\left\{\sum_{x=0}^{N-1} f(x) e^{-j((2 x+1) u \pi / 2 N)} .\right.
\end{aligned}
$$

It can be seen from the above formula that $\sum_{x=0}^{2 N-1} f e(x) e^{-j(2 x u \pi / 2 N)}$ is $2 N$ points, discrete Fourier transform, so in discrete cosine transform, you can extend the sequence length to $2 \mathrm{~N}$ and then make discrete Fourier transform, and the resulting result can be obtained by taking the real part to get the cosine transform. Then, the inverse transform can be expressed as follows:

$$
\begin{aligned}
F(0)= & \frac{1}{\sqrt{N}} F e(0)+\sqrt{\frac{2}{N}} \sum_{x=0}^{N-1} F e(u) \cos \frac{(2 x+1) u \pi}{2 N} \\
= & \frac{1}{\sqrt{N}} F e(0)+\sqrt{\frac{2}{N}} \operatorname{Re}\left\{\sum_{u=1}^{2 N-1} F e(u) e^{-j(2 x u \pi / 2 N)} e^{-j(u \pi / 2 N)}\right. \\
= & \left(\frac{1}{\sqrt{N}}-\sqrt{\frac{2}{N}}\right) F e(0) \\
& +\sqrt{\frac{2}{N}} \operatorname{Re}\left\{\sum_{u=0}^{2 N-1}\left[F e(u)(x) e^{-j(u \pi / 2 N)}\right] e^{-j(2 x u \pi / 2 N)}\right.
\end{aligned}
$$

As can be seen from the above formula, IDCT can be implemented by the $\mathrm{Fe}(u) e^{j \pi / 2 N}$ fast algorithm of IDN's $2 \mathrm{~N}$ point. When calculating the two-dimensional DCT transformation, the following calculation formula can be used to transform the two-dimensional DCT transformation into a one-dimensional DCT:

$$
\begin{gathered}
F(u, v)=\frac{1}{2} C(u)\left[\sum_{i=0}^{7} G(i, v) \cos \frac{(2 i+1) u \pi}{16}\right], \\
G(i, v)=\frac{1}{2} C(v)\left[\sum_{i=0}^{7} f(i, j) \cos \frac{(2 i+1) v \pi}{16}\right] .
\end{gathered}
$$


The starting point of this method is to perform DCT transformation on each small square of the data after decomposition. The main application is the dctmtx function in the image processing toolbox of MATLAB to return the DCT transformation matrix, and then the related processing is implemented.

2.3. Observation Indicators. Observation Index. All the subjects took $5 \mathrm{ml}$ of venous blood on an empty stomach in the morning. After the blood coagulated, they were centrifuged at $3000 \mathrm{r} / \mathrm{min}$ for $10 \mathrm{~min}$ to separate the serum and determine the LDL-C content in the serum within $2 \mathrm{~h}$. The test method is the direct determination of the Bissel test method. The two groups of patients were divided into 3 groups according to the results of LDL-C examination, namely, $2.7-3.4 \mathrm{mmol} / \mathrm{L}$ group, $3.4-4.2 \mathrm{mmol} / \mathrm{L}$ group, and $>4.2 \mathrm{mmol} / \mathrm{L}$ group. The MRS and DWI examination results of the two groups were compared, and the fat-water ratio was calculated as the area under the fat peak/(area under the water peak + area under the lipid peak) $\times 100 \%$, and the fatto-water ratio of the MRS in the three groups was compared in turn.

2.4. Statistical Analysis. SPSS18.0 statistical software is used for analysis. The chi-square test was used to compare ratios and analysis of variance for comparison of means. $P<0.05$ was considered statistically significant.

\section{Results}

3.1. Comparison of Clinical Features of NAFLD and NAFLD Combined with MS. The diagnostic criteria for no MS are only applicable to the NAFLD group, NAFLD combined MS group, and NAFLD combined group. Analyzing the variance of the clinical characteristics of the two groups, the results showed that age, BMI, SBP, DBP, TG, TC, LDL-C, HDL-C, FPG, ALT, AST, GGT, and UA differences were statistically significant $(P<0.01)$. After comparison, the BMI, SBP, DBP, TG, LDL-C, FPG, ALT, AST, GGT, and UA of the combined group and age group were all higher than those of the normal group $(P<0.01)$. HDL-C is lower than the control group $(P<0.01)$. In age combined group, BMI, SBP, DBP, TG, TC, LDL-C, FPG, ALT, AST, GGT, and UA were higher than those of group $(P<0.01)$, and low HDL-C ratio has a simple set of statistical significance $(P<0.01)$. There was no significant difference in TC between the normal group and the simple group $(P>0.05)$ (see Table 1$)[6]$.

3.2. Comparison of the Prevalence of NAFLD among Different Diagnostic Items in MS Diagnostic Criteria. Grouped according to the number of items that meet the MS4 diagnostic criteria, the results showed that the difference in prevalence among different groups was statistically significant $(P<0.01)$. The more items matched, the higher the prevalence of NAFLD. The prevalence of NAFLD in the 3 and 4 groups was higher than that in the 1 and 2 groups $(P<0.01)$. The prevalence of NAFLD in MS patients ( 3 items and 4 items) was $41.21 \%$.
3.3. DWI Results. According to the evaluation by two reviewers, the best DWI diagnosis $b$ value is $1000 \mathrm{~s} / \mathrm{mm}^{2}$. With the increase in $b$ value in the DWI image, the signals of various abdominal organs and lesions weaken to varying degrees, and the signals of normal organs weaken more evenly (Figures 3 and 4). Comparison of different $b$ values of nonalcoholic fatty liver and normal liver DWI is shown in Table 2.

3.4. MRS Results. The parameters of MRS in the observation group were significantly different from those in the control group $(P<0.01)$ (Table 3$)$.

3.5. Relationship between Magnetic Resonance and LDL-C. LDL-C level is as follows: 31 cases (39.74\%) in the $2.7-3.4 \mathrm{mmol} / \mathrm{L}$ group, fat-water ratio $(1.73 \pm 0.39) \% ; 26$ cases $(33.33 \%)$ in the $3.4-4.2 \mathrm{mmol} / \mathrm{L}$ group, fat-water ratio $(1.73 \pm 0.39) \%$; and $>4.2 \mathrm{mmol} / \mathrm{L}$ group of 21 cases $(26.92 \%)$, fat-water ratio $(13.77 \pm 6.17) \%$. Patients with a serum LDL-C of $2.7-3.4 \mathrm{mmol} / \mathrm{L}$ had a lipid-water ratio lower than $3.4-4.2 \mathrm{mmol} / \mathrm{L}(t=3.3197 ; P<0.01)$, and patients with a $3.4-4.2 \mathrm{mmol} / \mathrm{L}$ group had a lower lipid-water ratio $>4.2 \mathrm{mmol} / \mathrm{L}$ group $(t=6.7230 ; P<0.01)$.

\section{Discussion}

4.1. The Relationship between Nonalcoholic Fatty Liver and Metabolic Syndrome. NAFLD is a common clinical chronic liver disease, and progress is slow. Insulin resistance (IR) and excessive intake of high-calorie fatty substances are the most important factors that induce NAFLD. The incidence in Asia is about $12 \%-30 \%$. MS is a group of risk factors syndrome, including dyslipidaemia, hypertension, central obesity, and impaired glucose tolerance. In this study, a survey of 19,520 residents aged 20 to 80 in Chengdu showed that the prevalence of NAFLD was $12.33 \%$.

During the formation of NAFLD, overweight, type 2 diabetes or abnormal glucose metabolism, hyperlipidemia, and IR play an important role. The pathological basis of MS is central obesity and disorders of glucose and lipid metabolism, and IR is its central link. WHO's definition of MS includes IR, and it is a necessary condition for the diagnosis of MS. The results of this study showed that BMI, SBP, DBP, TG, TC, LDL-C, FPG and other MS-related indicators in the NAFLD group were higher than those of the normal group $(P<0.01)$, and HDL-C was lower than the normal group $(P<0.01)$; and with the increase in MS diagnosis items, the prevalence of NAFLD increases, indicating that the prevalence of NAFLD increases with the increase in metabolic disorders [7]. In this study sample, $41.21 \%$ of MS patients complicated with NAFLD, 28.13\% of NAFLD patients complicated with MS; and NAFLD combined with MS group ALT, AST, GGT, and other liver function indicators is higher than the NAFLD group alone, suggesting that NAFLD may be a metabolic disorder involving the liver clinical manifestations. 
TABLE 1: Comparison of clinical characteristics of patients with nonalcoholic fatty liver $(\bar{x} \pm s)$.

\begin{tabular}{|c|c|c|c|c|c|}
\hline Clinical features & Normal group & Simple NAFLD & NAFLD merges MS & $F$ value & $P$ value \\
\hline Age & $38.51 \pm 11.02 a b$ & $40.94 \pm 11.67$ & $49.95 \pm 12.67 a$ & 854.95 & $<0.01$ \\
\hline BMI & $21.32 \pm 1.99 a b$ & $23.36 \pm 1.31$ & $28.29 \pm 2.70 a$ & 5600.16 & $<0.01$ \\
\hline SBP & $109.22 \pm 10.90 a b$ & $112.88 \pm 10.55$ & $134.60 \pm 15.82 a$ & 1946.00 & $<0.01$ \\
\hline DBP & $71.33 \pm 7.50 a b$ & $74.33 \pm 7.36$ & $87.10 \pm 11.46 a$ & 1621.33 & $<0.01$ \\
\hline TG & $0.92 \pm 0.31 a b$ & $1.21 \pm 0.32$ & $3.10 \pm 2.08 a$ & 1957.34 & $<0.01$ \\
\hline TC & $4.53 \pm 0.82 a b$ & $4.63 \pm 0.80$ & $5.26 \pm 1.02 a$ & 529.81 & $<0.01$ \\
\hline LDL-C & $2.53 \pm 0.70 a b$ & $2.78 \pm 0.67$ & $3.06 \pm 0.87 a$ & 540.80 & $<0.01$ \\
\hline HDL-C & $1.69 \pm 0.38 a b$ & $1.42 \pm 0.31$ & $1.15 \pm 0.26 a$ & 1613.92 & $<0.01$ \\
\hline FPG & $4.92 \pm 0.40 a b$ & $5.04 \pm 0.47$ & $6.84 \pm 2.53 a$ & 950.13 & $<0.01$ \\
\hline ALT & $19.55 \pm 14.26 a b$ & $33.81 \pm 18.85$ & $44.75 \pm 29.05 a$ & 888.31 & $<0.01$ \\
\hline AST & $20.90 \pm 9.05 a b$ & $23.94 \pm 7.81$ & $30.18 \pm 14.27 a$ & 408.85 & $<0.01$ \\
\hline GGT & $18.13 \pm 17.54 a b$ & $30.05 \pm 29.42$ & $58.63 \pm 59.89 a$ & 615.86 & $<0.01$ \\
\hline UA & $292.22 \pm 71.38 a b$ & $366.94 \pm 79.00$ & $401.65 \pm 87.51 a$ & 1433.89 & $<0.01$ \\
\hline
\end{tabular}

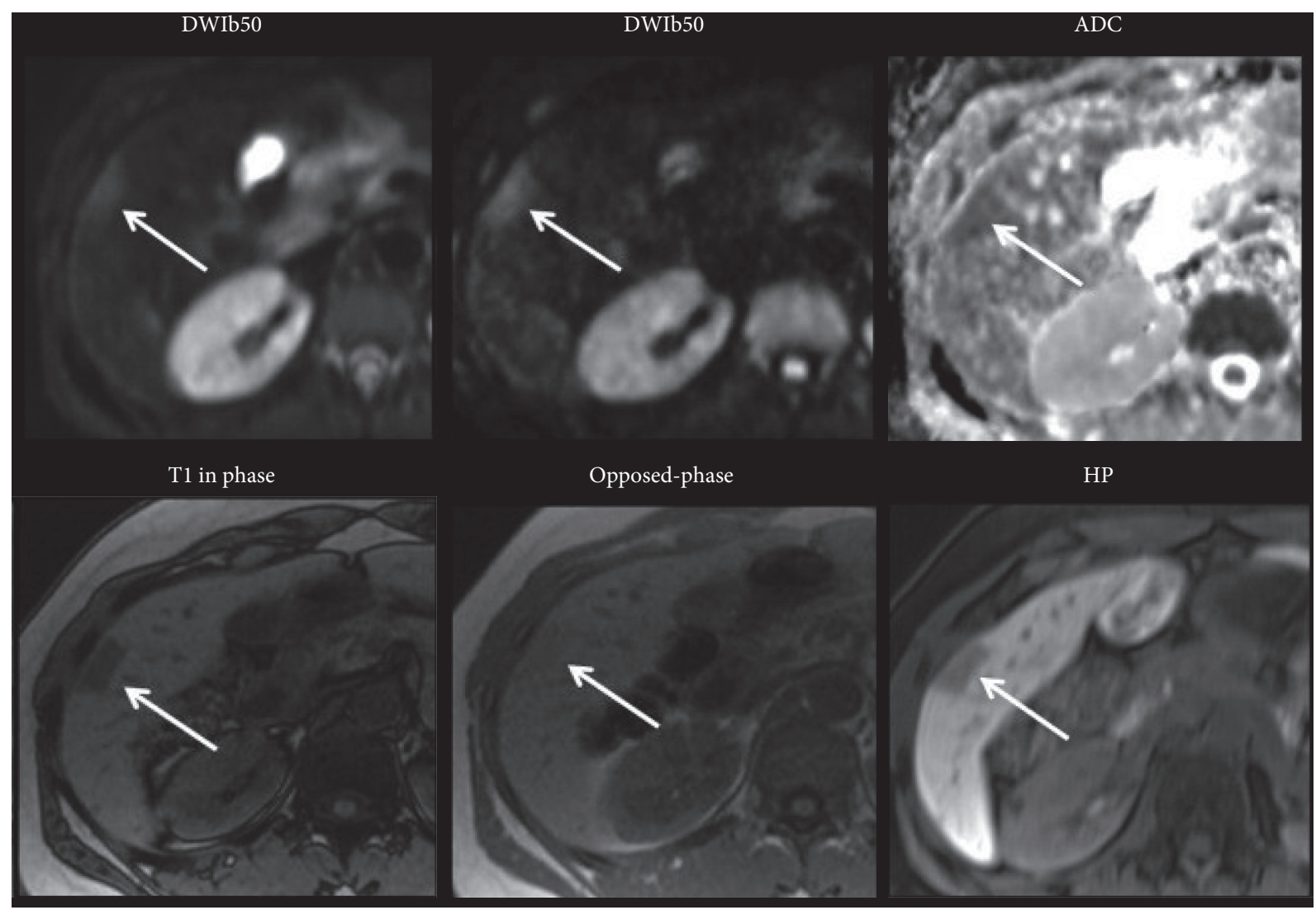

FIGURE 3: Normal liver DWI (control group).

4.2. Nonalcoholic Fatty Liver Magnetic Resonance DiffusionWeighted Imaging Knowledge. MRS is currently the only noninvasive medical imaging diagnosis method that reflects the pathophysiological changes of living tissues from a molecular level. It has a higher accuracy in the quantitative diagnosis of nonalcoholic fatty liver. In clinical studies, there are multiple spectrums of liver MRS, and the $1 \mathrm{H}$ spectrum is mostly used. In recent years, with the continuous improvement of MRI technology and the increasingly perfect software and hardware functions, the rapid imaging sequence of liver MRS is more and more widely used in clinical examinations. Self-breathing triggered MRS examination can effectively solve the interference of gastrointestinal gas and peristalsis to the examination results. The $1 \mathrm{H}-\mathrm{MRS}$ spectral line can clearly show the liver water peak height and area under the water peak, fat peak height, and area under the fat peak, and Cho peak display can be seen in some patients. When the body is in a high blood lipid environment, the fat synthesis rate of the liver is accelerated, and the rate of fat synthesis in the liver is significantly higher than that of low-density lipoprotein synthesis and secretion, which causes excess fat to accumulate in the liver, and its 


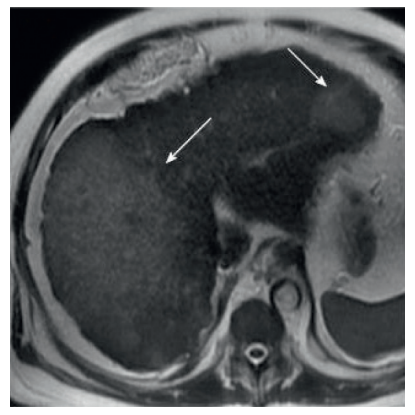

(a)

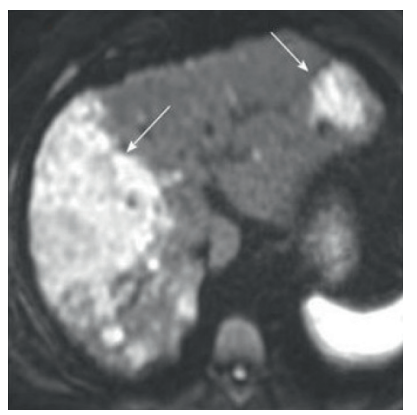

(d)

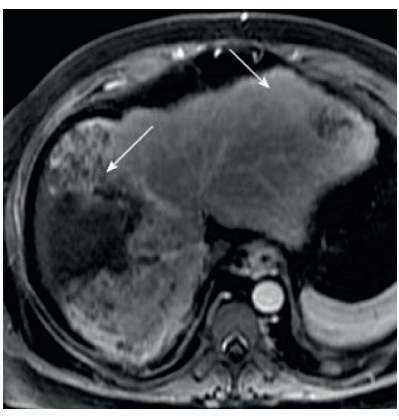

(b)

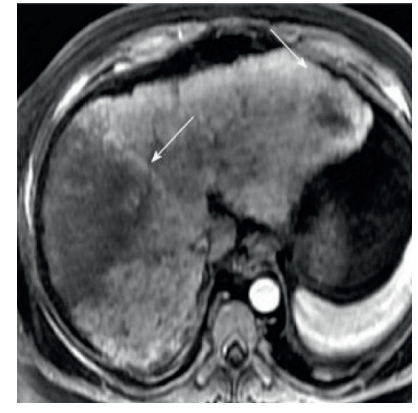

(c)

Figure 4: Nonalcoholic fatty liver DWI (observation group).

TABLE 2: Comparison of the reference value range of different $b$ values of nonalcoholic fatty liver and normal liver DWI.

\begin{tabular}{|c|c|c|c|c|c|}
\hline Group & $0 \mathrm{~s} / \mathrm{mm}^{2}$ & $50 \mathrm{~s} / \mathrm{mm}^{2}$ & $400 \mathrm{~s} / \mathrm{mm}^{2}$ & $800 \mathrm{~s} / \mathrm{mm}^{2}$ & $1000 \mathrm{~s} / \mathrm{mm}^{2}$ \\
\hline Control group $(n=39)$ & $1.81 \pm 0.19$ & $1.71 \pm 0.28$ & $1.55 \pm 0.23$ & $1.22 \pm 0.25$ & $1.15 \pm 0.21$ \\
\hline Observation group $(n=39)$ & $0.85 \pm 0.18^{*}$ & $0.81 \pm 0.12^{*}$ & $0.73 \pm 0.15^{*}$ & $0.64 \pm 0.11^{*}$ & $0.51 \pm 0.12^{\#}$ \\
\hline
\end{tabular}

TABLE 3: Comparison of various parameters of two sets of magnetic resonance MRS $(\bar{x} \pm s)$.

\begin{tabular}{lcccc}
\hline Group & Water peak height & Area under water peak & Fat peak height & Area under the fat peak \\
\hline Control group $(n=39)$ & $468.19 \pm 8.55$ & $158.23 \pm 5.23$ & $6.76 \pm 1.32$ & $3.53 \pm 1.01$ \\
Observation group $(n=39)$ & $391.19 \pm 8.90$ & $119.62 \pm 6.28$ & $23.16 \pm 4.94$ & $17.06 \pm 3.05$ \\
$T$ & 24.9554 & 17.0373 & 13.9896 & 17.3618 \\
$P$ & $<0.01$ & $<0.01$ & $<0.01$ & $<0.01$ \\
\hline
\end{tabular}

content is greater than $5 \%$. Sometimes, it can lead to the formation of fatty liver. $1 \mathrm{H}-\mathrm{MRS}$ calculates the relative content of intrahepatic fat by the area under the waveform, and the results are highly correlated with the pathological grade. DWI can provide accurate information on the water exchange function between tissue components and the composition of tissue space in the early physiological and pathological conditions. The ADC value of its dispersion coefficient can objectively and quantitatively reflect the pathological changes of tissues.

In addition, the measurement of different $b$ values of magnetic resonance DWI can also be used for quantitative analysis of nonalcoholic fatty liver. Magnetic resonance diffusion weighting and magnetic resonance spectroscopy can avoid shortcomings such as inability to quantify or have radiation damage. Qualitative and quantitative testing can provide objective indicators for clinical diagnosis and therapeutic evaluation. At the same time, it can be associated with a clinical test sensitivity index to evaluate DWI, the ability of $1 \mathrm{H}$-MRS to noninvasively and quantitatively diagnose the severity of nonalcoholic fatty liver disease [8].

\section{Conclusion}

In summary, NAFLD is closely related to MS, and the mutual aggravation and promotion of the two in the pathogenesis of the two should arouse enough attention. For patients with NAFLD, MS-related components should also be tested. In turn, active prevention and treatment of MS can reduce the incidence of NAFLD and slow the progression of NAFLD. This study shows that magnetic resonance MRS and DWI have significant differences between nonalcoholic fatty liver patients and normal patients. The DWI of the observation group mostly showed different degrees of 
uneven low signal, showing that there are typical multiple nodules and the signal is lower, and the normal liver DWI shows a more uniform low signal. The characteristics of the two are obvious, indicating that the magnetic resonance MRS and DWI are accurate and reliable in the diagnosis of nonalcoholic fatty liver. According to the LDL-C group comparison and magnetic resonance fat-water ratio, it is confirmed that the LDL-C test result is 2.7-3.4, and the MRS fat-water ratio is the lowest. LDL-C is closely related to magnetic resonance MRS and DWI. Magnetic resonance MRS and DWI provide a scientific basis for the clinical examination of nonalcoholic fatty liver.

\section{Data Availability}

The data used to support the findings of this study are available from the corresponding author upon request.

\section{Conflicts of Interest}

The authors declare that there are no conflicts of interest.

\section{Authors' Contributions}

Xiaoyu Liu and Zining Yao contributed equally to this work.

\section{Acknowledgments}

This work was supported by Hunan Province Clinical Medical Technology Innovation Guidance Project (2020SK52202) and Chenzhou Science and Technology Planning Project (jsyf2017020).

\section{References}

[1] Z. Mousavi, A. Ganji, D. Farrokh Tehrani, A. Bahari, A. Esmaeil Zadeh, and M. Delghandi, "Correlation of visfatin level with non-alcoholic fatty liver in metabolic syndrome," Medical Journal of the Islamic Republic of Iran, vol. 31, no. 1, 28 pages, 2017.

[2] R. Gaharwar, S. Trikha, S. L. Margekar, O. P. Jatav, and P. D. Ganga, "Study of clinical profile of patients of non alcoholic fatty liver disease and its association with metabolic syndrome," Journal of the Association of Physicians of India, vol. 63, no. 1, pp. 12-16, 2015.

[3] H. Uzun, "Comment on "relationship between non-alcoholic fatty liver disease and benign prostatic hyperplasia/lower urinary tract symptoms: new insights from an Italian crosssectional study"' World Journal of Urology, vol. 33, no. 5, 753 pages, 2015.

[4] L. R. Cuenza, T. L. J. Razon, and J. C. Dayrit, "Correlation between severity of ultrasonographic nonalcoholic fatty liver disease and cardiometabolic risk among Filipino wellness patients," Journal of Cardiovascular and Thoracic Research, vol. 9, no. 2, pp. 85-89, 2017.

[5] L. Papalavrentios, E. Sinakos, D Chourmouzi et al., "Value of 3 tesla diffusion-weighted magnetic resonance imaging for assessing liver fibrosis," Annals of Gastroenterology, vol. 28, no. 1, pp. 118-123, 2015.

[6] D. Crisp, H. R. Pollock, R. Rosenberg et al., "The on-orbit performance of the orbiting carbon observatory-2 (oco-2) instrument and its radiometrically calibrated products,"
Atmospheric Measurement Techniques, vol. 10, no. 1, pp. 59-81, 2017.

[7] X. Cheng, M. D. Ding, and C. Fang, "Imaging and spectroscopic diagnostics on the formation of two magnetic flux ropes revealed by sdo/aia and iris," The Astrophysical Journal, vol. 804, no. 2, pp. 1433-1440, 2015.

[8] R. Jin, X. Li, Y. Yan et al., "Detection method of multi-target recognition of potato based on fusion of hyperspectral imaging and spectral information," Transactions of the Chinese Society of Agricultural Engineering, vol. 31, no. 16, pp. 258-263, 2015. 